\title{
Comparison of palatal bone height in different facial morphological patterns by cone beam computed tomography
}

\author{
Gabriella Lopes de Rezende Barbosa ${ }^{1}$, Laura Ricardina Ramirez-Sotelo ${ }^{1}$, \\ Phillipe Nogueira Barbosa Alencar ${ }^{1}$, Solange Maria de Almeida ${ }^{1}$
}

${ }^{1}$ Universidade Estadual de Campinas - UNICAMP, Piracicaba Dental School, Department of Oral Diagnosis, Area of Oral Radiology, Piracicaba, SP, Brazil

\begin{abstract}
Aim: To evaluate the relationship between palatal bone height and facial types using cone beam computed tomography (CBCT) images. Methods: The study sample consisted of $110 \mathrm{CBCT}$ images. Subjects aged 18 to 35 years old were classified as brachyfacial, mesofacial and dolichofacial, according to SN.GoGn angle, measured in cephalometric analysis with Nemotec $3 \mathrm{D}$ software. Three observers evaluated the bone height of the palate in the anterior region, at the level of the maxillary first premolars. The measurements were performed in sagittal and coronal views, in median and paramedian regions of the palate, also using Nemotec 3D software. Measurements were repeated after 15 days on $25 \%$ of the sample to analyse intra and interobserver agreements by $\mathrm{CCl}$ test. Analysis of variance was performed to calculate statistical differences between the bone heights of the three facial types, with a significance level of $5 \%$. Results: The correlation level of intra-observer agreement was excellent. No significant differences were observed between the palatal bone height in the region of first premolars and the different facial types ( $p>0.05)$. Conclusions: Based on CBCT evaluations, there is no association between palatal bone height and facial morphological patterns.
\end{abstract}

Keywords: cone-beam computed tomography; diagnostic imaging; orthodontic anchorage procedures.

\section{Introduction}

The facial type of an individual comprises variations of the craniofacial skeleton structures and it is related to genetic and environmental factors ${ }^{1-2}$. The classification of facial types has a direct relationship with craniofacial growth and is divided in three types: brachyfacial, mesofacial and dolichofacial. In the brachyfacial type, there is a tendency to horizontal facial growth; mesofacial type is characterized by balanced growth of all facial thirds and dolichofacial type has a tendency to vertical facial growth and mouth breathing ${ }^{3-4}$.

These facial types are related to aesthetics and functional features of bones and muscles. They also present significant correlation with dental arch features, which can lead to other complex orofacial changes ${ }^{1,5-6}$.

The proper determination of variations in facial types is important for orthodontic diagnosis and treatment since certain procedures performed during orthodontic therapy may attenuate or enhance facial features. Morphological changes can occur in several structures such as the hard palate, a structure of the 
maxillofacial complex that is involved anatomically and functionally in all stages of the craniofacial development ${ }^{7}$.

According to the facial type, the palate can be, for example, deep and narrow in the dolichofacial individuals while wide and shallow in brachyfacial subjects ${ }^{8-12}$.

According to Christie $^{13}$, features of each facial type may have a significant implication in orthodontic treatment planning, as well as the configuration of dental arches and amount of available bone. This individualized approach allows an adaptation of the orthodontic mechanics, containment procedures and in some cases, even skeletal anchorage procedures allowing greater control of the results ${ }^{14}$.

The amount of available bone in regions for insertion of anchorage implants is a limiting factor for orthodontic treatments that require skeletal anchorage, especially in cases of palatal mini-implants. Even temporary, such implants require a satisfactory bone volume for placement and stability, and must be inserted at safe distances from vital structures that cannot be injured ${ }^{14-16}$. Since the region of first premolars has the highest levels of bone height and most clinically satisfactory palatal implants are inserted at this level, this was the site selected for evaluation in the present study ${ }^{14,17}$.

Several authors compared the dimensions of the hard palate among facial types by clinical evaluations and plaster model analysis ${ }^{2,18-20}$. However, in general, such studies assess characteristics like the morphology of palatal vault, palatal depth and thickness. In this sense, few of them have emphasized by quantitative measurements the internal assessment of the palate such the bone height.

The study conducted by Esteves and Bommarito ${ }^{21}$ (2007) proved the correlation of palatal depth and facial type, leading to the authors' hypothesis that other palatal features would also be influenced by the facial type.

Taking into account the lack of reports concerning the relationship between facial types and palatal bone height, and the raised hypothesis, this research aimed to evaluate the relationship between palatal height and the different facial types, brachyfacial, mesofacial and dolichofacial, using cone beam computed tomography (CBCT) images. Moreover, a positive correlation would lead to a possible predictability of the bone amount in the palatal region of patients classified with a determined facial pattern.

\section{Material and methods}

After the approval of this study by the Ethics Committee in Research (protocol number 118/2012), 110 CBCT scans were selected from an archive. The sample consisted of images from patients (18-35-year-old) with no evident facial and dental asymmetries, torus palatinus or anomalies in the head and neck region. The patients had complete permanent dentition and were referred to the tomographic examination for different treatment purposes unrelated to this study. CBCT scans were obtained by an i-CAT unit (Imaging Sciences International, Inc., Pennsylvania, USA) with the following parameters: voxel size of $0.3 \mathrm{~mm}, 120 \mathrm{kVp}, 8 \mathrm{~mA}$, FOV of 23 x $17 \mathrm{~cm}$, and $40 \mathrm{~s}$ scan time. The assessment of the images was done in a secluded room with dim light by expert oral radiologists

First, to classify each subject according to the facial type, RaySum multi-planar reformations were created using Nemotec software (Nemoceph Imaging Cephalometric and Tracing Software S.L., Spain), based on the CBCT volume. This reformatted image, also known as virtual lateral radiograph (VLR), simulates a two-dimensional lateral exam, enabling the acquisition of cephalometric analysis on this exam. The facial type was determined based on the SN.GoGn angle that was measured by one of the authors on the VLR images through the computerized module Nemoceph 2D in Nemotec software (Nemoceph Imaging Cephalometric and Tracing Software S.L., Spain). According to the obtained SN.GoGn angle, the subjects were classified according to the following criteria: brachyfacial, $\leq 27^{\circ}$; mesofacial, between $27^{\circ}$ and $37^{\circ}$ and dolichofacial, SN.GoGn $\geq 37^{\circ 22}$.

After determining the facial type of the sample, the amount of available bone was assessed by three expert oral radiologists with at least two years experience in tomographic appraisal. Using Nemoceph 3D module (Nemoceph Imaging Cephalometric and Tracing Software S.L., Spain) the bone height in the anterior region of the hard palate was assessed at the level of the maxillary first premolars.

The regions of interest were determined in multi-planar reconstructions (MPR), using the orientation lines of the planes. First, in the axial view, the mid-sagittal plane of the patient and the maxillary first premolar region were defined, superimposing the sagittal and coronal lines, respectively (Figure 1A). The midsagittal plane was created by an imaginary line perpendicular to the Frankfurt plane that divided the body into right and left halves oriented by the anterior nasal spine. The maxillary first premolar region was defined by the plane that overlaps the root canals of both right and left first premolars.

For bone height assessment, linear measurements were performed in sagittal and coronal planes, using the ruler tool of the software, creating measures superimposed to the orientation line of the planes, predetermined on the axial view as described before.

In the sagittal view, the measurement was performed between the outer cortical layer of the nasal floor and the cortical layer of the oral hard palate, superimposed to the coronal orientation line (Figure 1B). Next, in the coronal view, the palatal bone height of the median and paramedian regions were assessed. The median/central site was assessed superimposing the measurement on the orientation line of the sagittal plane. Then, the paramedian sites were defined and evaluated $3 \mathrm{~mm}$ and $6 \mathrm{~mm}$ bilaterally to the central measurement (Figure 1C).

After one week interval, the same evaluation was repeated with $25 \%$ of the sample to evaluate statistically the intra-observer agreement. The intra- and inter-observer agreements were evaluated by the Intraclass Correlation Coefficient (ICC) using SPSS $^{\circledR}$ (Statistical Package for Social Sciences) program package version 17.0 (SPSS Inc., Chicago, IL, USA) for Windows. 


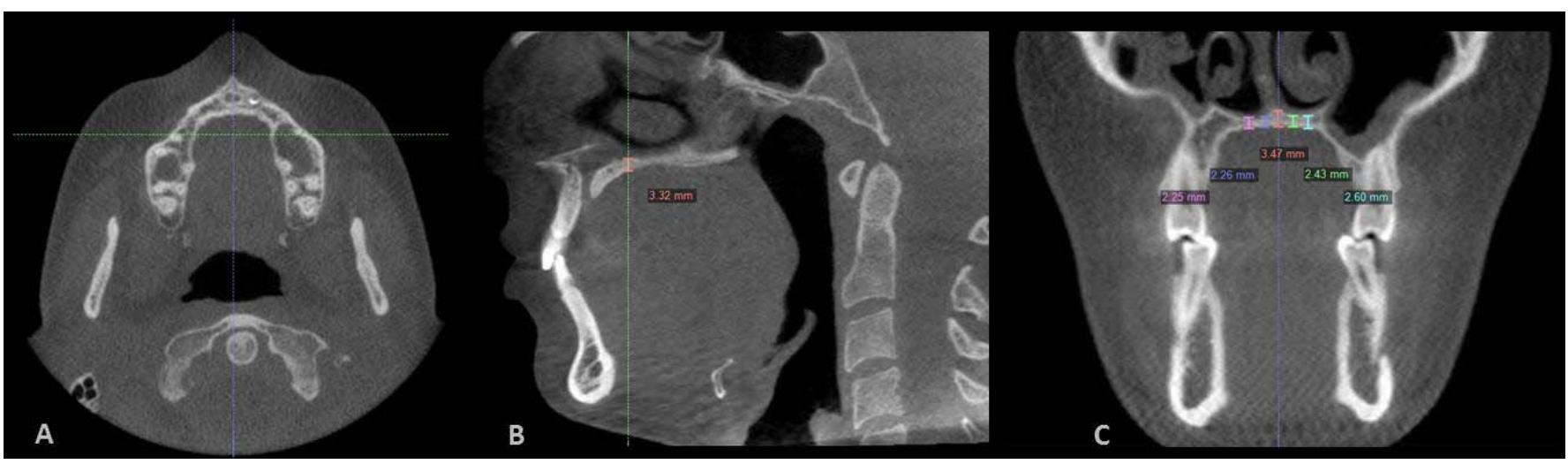

Fig. 1. Palatal bone height evaluation in multiplanar reconstruction of CBCT image. Definition of mid-sagittal plane and upper first premolar region (A). Linear measurement corresponding to midsagittal palatal bone height superimposed on the orientation line - sagittal view (B). Median and paramedian measurements $3 \mathrm{~mm}$ and $6 \mathrm{~mm}$ bilaterally - coronal view (C).

The means of the measurements obtained by the three examiners were calculated.

The mean of the values obtained in the coronal view (median and paramedian sites) were also obtained. ANOVA was performed to calculate the statistical differences between the bone heights from the three different facial types, with a significance level of 5\%, using SAS statistical package version 20.0 (SAS Inc., Cary, NC, USA) for Windows.

\section{Results}

One hundred and ten images from patients aged 18 to 35 years comprised the final sample of the study. The distribution of sample groups was similar in all three divisions: 38 brachyfacial subjects, 38 mesofacial and 34 dolichofacial individuals. Regarding the correlation level of the intra-observer agreement according to the intra-class correlation coefficient, the values were close to 1 for the three examiners, which represents an excellent correlation, as interpreted by Szklo and Nieto ${ }^{23}$ (2000).

The comparison of mean values for palatal bone height in different facial types, according to the analysis of variance, showed no statistically significant differences between the groups in both evaluations, sagittal $(p=0.738)$ and coronal assessments $(p=0.695)$ (Table 1$)$.

\section{Discussion}

The influence of the facial type on functional, neuromuscular, skeletal and dental functions has been subject

Table 1. Mean and standard deviation (SD) values for palatal bone height of the evaluated facial patterns in midsagittal and coronal views $(\mathrm{mm})$

\begin{tabular}{lllll}
\hline Facial type & \multicolumn{2}{l}{ Midsagittal view } & \multicolumn{2}{l}{ Coronal view } \\
& Mean & SD & Mean & SD \\
Brachyfacial & 7.32 & 2.73 & 7.22 & 2.35 \\
Dolichofacial & 6.82 & 2.72 & 7.07 & 2.91 \\
Mesofacial & 6.97 & 2.59 & 6.78 & 2.07 \\
\hline
\end{tabular}

No statistical difference between the groups. of debate in articles, texts and researches for over a century ${ }^{24}$. The evaluation of craniofacial characteristics and morphology are usually made by conventional lateral radiographs $s^{7,9}$, which are believed by several authors to be effective for the diagnosis and orthodontic planning ${ }^{24}$. However, recent threedimensional imaging technologies, such as $\mathrm{CBCT}$, allow the assessments of craniofacial structures without some disadvantages of the conventional techniques, especially the superimposition of structures ${ }^{25}$.

According to Cortes et al., 2012, CBCT is an accurate image-acquisition method to provide information regarding bone measurements, which are important, for example, to perform precise surgical planning for implant placement ${ }^{26}$. Taking into account this advantage and the improvement in the visualization of the palatal region anatomy, this study was conducted based on tomographic images. This threedimensional approach allowed the accurate quantitative evaluation of internal features of the region, different from previous studies that assessed distinct features such as the palatal morphology using plaster models ${ }^{2,18-20}$.

The facial typology is given according to anatomical variations that form the craniofacial complex ${ }^{27}$. Establishing the facial pattern of each patient and fit them into a classification is required since each facial type presents different morphological and functional features ${ }^{4}$.

The growth trend of the thirds of the face determines the patient's facial type and the direction of its evolution, which can be more horizontal (brachyfacial), balanced (mesofacial) or vertical (dolichofacial) ${ }^{3}$. In this sense, the shape of the palate is one of the individual characteristics that are subject to influence of the facial typology and may have different morphologies ${ }^{21}$.

Assessment of the palatal bone height in cases of skeletal anchorage using palatal mini-implants is the key to indicate or contraindicate the procedure, also preventing possible injuries to structures adjacent to implant insertion sites, such as perforation of the nasal floor ${ }^{15,28}$.

Based on our results, there were no significant differences between the palatal measurements evaluated in the three different facial patterns. Thus, it is not possible to correlate palatal bone height with facial types and neither 
estimate a larger amount of bone in this region as a predictor for surgical procedures.

There is lack of studies in the literature that aimed to evaluate bone availability of the palate, as well as to correlate this information with variations in the maxillofacial complex. In this sense, our results can only be confronted with studies that had different approaches but also evaluated the palate according to facial types.

The results of the present study disagree with Esteves and Bommarito reports ${ }^{21}$ (2007), who stated that dolichofacial individuals have narrow and deep palate while brachyfacial patients have a wider and shallow palate, demonstrating differences in the palatal morphology in each facial type, an outcome not observed in our study. However, these characteristics were assessed in plastic models, disagreeing with our methodology and the selected palatal feature.

Our results also differ from previous findings of significant differences between the depths of the palate according to facial types. Despite addressing different characteristics, it is believed that in the same way that the depth evaluated in plaster models varies according to the facial pattern, the height palate would also suffer such influences ${ }^{19}$. However, the lack of correlation between the palatal heights found in the present study disagrees with these findings.

The present study evaluated bone height in two different views of the tomographic multiplanar reconstruction in order to avoid the interference of anatomical structures in the midsagittal view. The evaluation was then performed in different sites of the same region to provide additional values, since it is proven that paramedian sites have comparable heights when compared to median measurements ${ }^{29}$.

Based on CBCT evaluations, it may be concluded that there is no association between palatal bone height and facial patterns. According to the obtained data, it may be observed that there is no predictability of palatal bone height and it is therefore recommended to request appropriate diagnostic imaging modality for planning interventions in the region of the hard palate, reducing the risk of injuries and perforations.

\section{References}

1. Cassidy KM, Harris EF, Tolley EA, Keim RG. Genetic influence on dental arch form in orthodontic patients. Angle Orthod. 1998; 68: 445-54.

2. Retamoso LB, Knop LA, Guariza Filho O, Tanaka OM. Facial and dental alterations according to the breathing pattern. J Appl Oral Sci. 2011; 19: $175-81$.

3. McNamara JA. Influence of respiratory pattern on craniofacial growth. Angle Orthod. 1981; 51: 269-300.

4. Kageyasma T, Domínguez-Rodríguez GC, Vigorito JW, Deguchi T. A morphological study of the relationship between arch dimensions and craniofacial structures in adolescents with Class II Division 1 malocclusions and various facial types. Am J Orthod Dentofacial Orthop. 2006; 129: 368-75.

5. Osburn RC. The effects of brachycephaly and dolichocephaly on the teeth of man. Dent Cosmos. 1910; 52: 517-26.

6. Bhat M, Enlow DH. Facial variations related to headform type. Angle Orthod. 1985; 55: 269-80.
7. Faria PT, de Oliveira Ruellas AC, Matsumoto MA, Anselmo-Lima WT, Pereira FC. Dentofacial morphology of mouth breathing children. Braz Dent J. 2002; 13: 129-32.

8. Linder-Aronson $\mathrm{S}$. The relation between nasorespiratory function and dentofacial morphology. Am J Orthod. 1983; 83: 443-4.

9. Frasson JM, Magnani MB, Nouer DF, de Siqueira VC, Lunardi N. Comparative cephalometric study between nasal and predominantly mouth breathers. Braz J Otorhinolaryngol. 2006; 72: 72-81.

10. Bianchini AP, Guedes ZC, Vieira MM. A study on relationship between mouth breathing and facial morphological pattern. Braz J Otorhinolaryngol. 2007; 73: 500-5

11. Cattoni DM, Fernandes FD, Di Francesco RC, Latorre Mdo R. Characteristics of the stomatognathic system of mouth breathing children: anthroposcopic approach. Pro Fono. 2007; 19: 347-51.

12. Coelho AR, Tanaka O, Ribeiro JS, Machado MA, Camargo ES. Transverse craniofacial dimensions in Angle Class II, Division 1 malocclusion according to breathing mode. Braz Oral Res. 2010; 24: 70-5.

13. Christie TE. Cephalometric patterns of adults with normal occlusion. Angle Orthod. 1977; 47: 128-35.

14. Cousley R. Critical aspects in the use of orthodontic palatal implants. Am J Orthod Dentofacial Orthop. 2005; 127: 723-9.

15. Wehrbein $\mathrm{H}$, Feifel $\mathrm{H}$, Diedrich P. Palatal implant anchorage reinforcement of posterior teeth: A prospective study. Am J Orthod Dentofacial Orthop. 1999; 116: 678-86.

16. Feldmann I, Bondemark L. Orthodontic anchorage: a systematic review. Angle Orthod. 2006; 76: 493-501.

17. Jung $B A$, Wehrbein $H$, Heuser $L$, Kunkel $M$. Vertical palatal bone dimensions on lateral cephalometry and cone-beam computed tomography: implications for palatal implant placement. Clin Oral Implants Res. 2011; 22: 664-8.

18. Braun S, Hnat WP, Fender DE, Legan HL. The form of the human dental arch. Angle Orthod. 1998; 68: 29-36.

19. De Freitas FCN, Bastos EP, Primo LS, de Freitas VL. Evaluation of the palate dimensions of patients with perennial allergic rhinitis Int J Paediatr Dent. 2001; 11: 365-71.

20. Armando RS, Bommarito S, Mandetta S, Zanelato ACT. Evaluation of palatine depth in patients with different facial types. Rev. Odonto Cienc. 2002; 10: 15-22.

21. Esteves A, Bommarito S. Evaluation of palatine depth and dimensions of the upper dental arch in patients with malocclusion and different facial types. R Dental Press Ortodon Ortop Facial. 2007; 12: 84-98.

22. Riedel RA. The relation of maxillary structures to cranium in malocclusion and normal occlusion. Angle Orthod. 1952; 22: 142-5.

23. Szklo R, Nieto FJ. Epidemiology beyond the basics. 2nd ed. Gaithersburg: Aspen Publishers; 2000. p.343-404.

24. Kluemper GT, Vig PS, Vig KW. Nasorespiratory characteristics and craniofacial morphology. Eur J Orthod. 1995; 17: 491-5.

25. Lascala CA, Panella J, Marques MM. Analysis of the accuracy of linear measurements obtained by cone beam computed tomography (CBCTNewTom). Dentomaxillofac Radiol. 2004; 33: 291-4.

26. Cortes ARG, Gomes AFAM, Tucunduva MJAPS, Arita, ES. Evaluation of linear tomography and cone beam computed tomography accuracy in measuring ridge bone width for planning implant placement. $\mathrm{Br} \mathrm{J}$ Oral Sci. 2012; 11: 116-9.

27. Sies ML, Farias SR, Vieira MM. Oral breathing: relationship between facial type and dental occlusion in adolescents. Rev Soc Bras Fonoaudiol. 2007; 12: 191-8.

28. Cousley RR, Parberry DJ. Combined cephalometric and stent planning for palatal implants. J Orthod. 2005; 32: 20-5.

29. de Rezende Barbosa GL, Ramírez-Sotelo LR, Távora DM, Almeida SM. Comparison of median and paramedian regions for planning palatal miniimplants: a study in vivo using cone beam computed tomography. Int J Oral Maxillofac Surg. 2014; 43: 1265-8. 